\title{
Calibration of a high spatial resolution laser two-color heterodyne interferometer for density profile measurements in the TJ-II stellarator ${ }^{a)}$
}

\author{
Pablo Acedo, ${ }^{1}$ P. Pedreira, ${ }^{1}$ A. R. Criado, ${ }^{1}$ Horacio Lamela, ${ }^{1}$ Miguel Sánchez, ${ }^{2}$ and \\ Joaquín Sánchez ${ }^{2}$ \\ ${ }^{1}$ Universidad Carlos III de Madrid, Avda. de la Universidad 30, Leganés, 28911 Madrid, Spain \\ ${ }^{2}$ Laboratorio Nacional de Fusión por Confinamiento Magnético-CIEMAT, Ciudad Universitaria s/n, \\ 28040 Madrid, Spain
}

(Presented 13 May 2008; received 12 May 2008; accepted 30 July 2008; published online 31 October 2008)

\begin{abstract}
A high spatial resolution two-color $\left(\mathrm{CO}_{2}, \lambda=10.6 \mu \mathrm{m}, \mathrm{He}-\mathrm{Ne}, \lambda=633 \mathrm{~nm}\right)$ interferometer for density profile measurements in the TJ-II stellarator is under development and installation, based in the currently operational single channel two-color heterodyne interferometer. To achieve the objectives of 32 channels, with $4-5 \mathrm{~mm}$ lateral separation between plasma chords, careful design and calibration of the interferometric waveforms for both the measurement and vibration compensation wavelengths are undertaken. The first step has been to set up in our laboratories an expanded-beam heterodyne/homodyne interferometer to evaluate the quality of both interferometric wavefronts, a reported source of poor vibration compensation and thus low resolution in the density profile measurements. This novel interferometric setup has allowed us to calibrate the spatial resolution in the profile measurements resulting in $\sim 2 \mathrm{~mm}$ lateral resolution in the reconstruction of the interferometric wavefront. (C) 2008 American Institute of Physics. [DOI: 10.1063/1.2973326]
\end{abstract}

\section{INTRODUCTION}

Laser two-color heterodyne interferometric systems are a proven diagnostic to measure electron density in fusion plasmas, ${ }^{1}$ and are now operational in several tokamaks ${ }^{2}$ and stellarators. ${ }^{3}$ Some of these systems incorporate several measurement channels over different plasma views to recover the temporal evolution of the density profile during discharges. ${ }^{4}$ In the past years we have witnessed an increasing interest in the development of interferometric density profile measurement systems with high spatial resolution for short scalelength $(\sim 1-5 \mathrm{~mm})$ density fluctuation studies. ${ }^{5}$ To achieve such a high spatial resolution, far infrared (FIR) wavelengths are no longer valid ${ }^{6}$ and different solutions have been proposed including those based on frequency doubled diode pumped lasers ${ }^{5}$ and yttrium aluminum garnet/ $/ \mathrm{CO}_{2}$ systems. ${ }^{7}$ Nevertheless, in all the cases, mechanical vibration compensation appears as the main issue in order to obtain such high spatial resolutions, as the residual mechanical noise (uncompensated vibration) degrades the measurements. ${ }^{5,7}$ Poor wavefront quality, especially for the smaller wavelength, has been identified as a major source of such error, ${ }^{7}$ and a systematic study of the influence of the quality of the interferometric wavefront is thus crucial to achieve the abovementioned spatial resolutions. Besides this, the evaluation of the ultimate spatial resolution in the profile reconstruction in the $1 \mathrm{~mm}$ scale is difficult.

We are currently involved in the design and installation of a high spatial resolution two-color $\left(\mathrm{CO}_{2}, \lambda=10.6 \mu \mathrm{m}\right.$, $\mathrm{He}-\mathrm{Ne}, \lambda=633 \mathrm{~nm}$ ) interferometer for density profile mea-

\footnotetext{
a) Contributed paper, published as part of the Proceedings of the 17th Topical Conference on High-Temperature Plasma Diagnostics, Albuquerque, New Mexico, May 2008.
}

surements in the TJ-II Stellarator, ${ }^{8}$ based on the currently operational single channel two-color heterodyne interferometer. ${ }^{3}$ To achieve the objectives of 32 channels, $4-5 \mathrm{~mm}$ lateral resolution, careful design and calibration of the interferometric waveforms for both the measurement and vibration compensation wavelengths have to be undertaken. For this reason, a novel expanded-beam heterodyne/homodyne interferometer has been designed to set up a methodology for complete evaluation of the quality of both interferometric wavefronts in high spatial resolution two-color systems and to evaluate the actual spatial resolution of such systems. This optical scheme has allowed us to calibrate the temporal and spatial evolution of a heterodyne interferometric wavefront versus a standard homodyne interferometer, and helped us to evaluate the different optical and electronic components to be incorporated in the final diagnostic for the stellarator TJII. We also evaluated the optical and electrical cross-talk between adjacent channels, as this has also been reported to be a major source of error in these diagnostics. ${ }^{9}$ In the present work we focus on the results obtained with such scheme on heterodyne interferometric profile reconstruction and its calibration against the reference homodyne wavefront and the evaluation of the spatial resolution obtained for the heterodyne system.

\section{EXPANDED-BEAM HETERODYNE/HOMODYNE INTERFEROMETER FOR INTERFEROMETRIC WAVEFRONT CALIBRATION}

Figure 1 shows the optical layout of the expanded-beam heterodyne/homodyne interferometer for interferometric wavefront calibration based on a heterodyne/homodyne system used by our group for precise calibration of the heterodyne phase measurement $\left(10^{-3}\right.$ of a fringe) against the ho- 


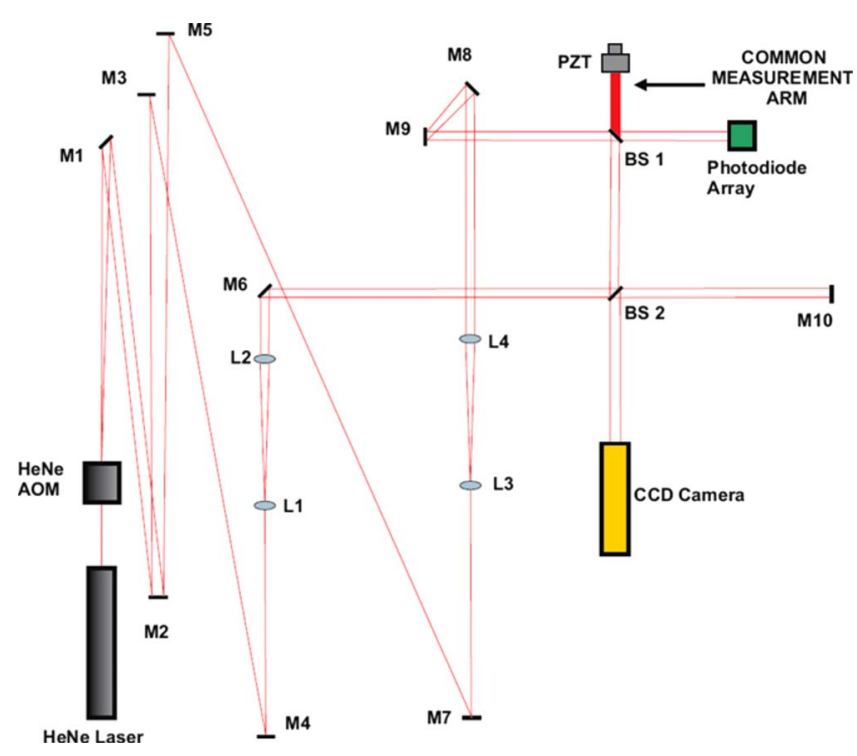

FIG. 1. (Color online) Layout of the expanded-beam heterodyne/homodyne interferometer for interferometric wavefront calibration. Note the common measurement arm (see text for details).

modyne interferometer for the single channel plasma density diagnostic of the stellarator TJ-II. ${ }^{10}$ The motivation for this optical scheme is the inherent difficulty to evaluate the heterodyne interferometric wavefront quality and the actual spatial resolution of the profile reconstruction. In these diagnostics the phase is measured independently for each point (detector) and the spatial profile reconstructed point to point, and the influence of optical elements as the modulators and mirrors in the wavefront are no easily identified. In front of this, standard homodyne interferometry allows a direct and continuous measurement of the interferometric wavefront through the evaluation of the light intensity phase pattern and the evaluation of the quality of the interferences in terms of visibility. In this framework, this expanded-beam heterodyne/homodyne interferometer will allow us to evaluate the quality and spatial resolution in the measurement and reconstruction of the heterodyne phase front using the homodyne as a reference.

The system shown in Fig. 1, installed in the laboratories of the group, consists of two interferometers, a heterodyne Mach-Zehnder and a homodyne Michelson which share the same measurement arm (common measurement arm in Fig. 1) for interferometric phasefront comparison. The length of this common arm is controlled by a piezoelectric transducer (PZT) (Fig. 1) to study the evolution of the wavefronts under a known stimulus. This system works as follows. The output of a $5 \mathrm{~mW} \mathrm{He}-\mathrm{Ne}$ laser $(633 \mathrm{~nm})$, used as a light source, enters an acousto-optic modulator. The incoming light beam is then split into the zeroth and first orders, acting as a beamsplitter that introduces a frequency shift between both beams for the heterodyne system. Both orders are then expanded with a telescopic system to a $10 \mathrm{~mm}$ diameter beams that enter the Michelson scheme (reference arm: BS2, M10, BS2; measurement arm: BS2, PZT, BS2) and the Mach-Zehnder scheme (measurement arm: M6, BS2, PZT, BS1; reference arm: M8, M9, BS1). Detection of the homodyne interferometric waveform is carried out with a charge coupled device

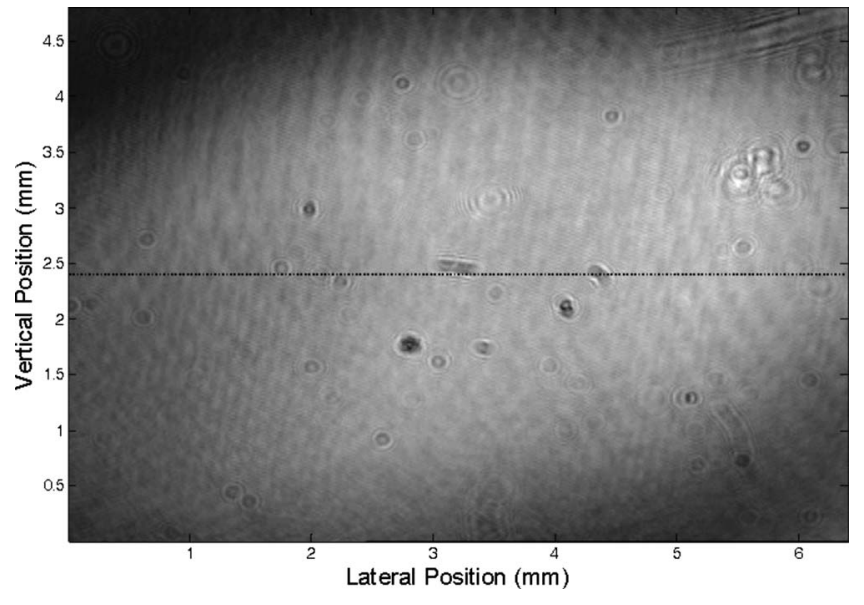

FIG. 2. Homodyne interferometric pattern as recovered with the CCD camera. Horizontal line: projection of the photodiode array for the heterodyne system.

(CCD) camera (6.4 mm sensor width, $768 \times 494$ pixels), while a 35 elements photodide array $(1 \mathrm{~mm}$ center separation) is used for the heterodyne signal. As the size of the CCD detector is $6.4 \mathrm{~mm}$, we have restricted our study to such wavefront dimensions. The idea is to avoid any extra optical elements (i.e., a beam expander) that could modify the wavefront besides the stimulus and changes introduced in the common path of the interferometer. We have restricted our study to the He-Ne wavelength as the wavefront quality of the smaller wavelength in $\mathrm{CO}_{2} / \mathrm{He}-\mathrm{Ne}$ plasma density diagnostics is the major source of poor vibration compensation in high spatial resolution interferometric measurements. ${ }^{7}$

\section{INTERFEROMETRIC HETERODYNE WAVEFRONT CHARACTERIZATION USING A HETERODYNE/ HOMODYNE INTERFEROMETER}

Figure 2 shows a typical homodyne interferometric pattern for the expanded-beam interferometer (CCD detector). The shown interferometric pattern corresponds to a "flat" phase profile as all the points in the wavefront are in the same fringe and the phase difference between all of them (path-length difference) is less than a wavelength. Note in Fig. 2 the line drawn in the projection of the heterodyne detector (photodiode array) in the CCD camera (Fig. 1). The phase profile along this line will be used as the reference.

In this situation we introduce a stimulus to the PZT in order to change the pathlength of the common arm of the interferometric system. In this case both wavefronts are to suffer the same longitudinal variation in pathlength difference while the spatial profiles are to remain invariant. In Fig. 3 we show the stimulus and the recovered temporal evolution for the phase obtained in three channels (out of seven) for the heterodyne detector (1 $\mathrm{mm}$ spatial separation).

In Fig. 4 we show the heterodyne interferometric profile reconstructed from the signals obtained from the different detectors (Fig. 3) at two different instants of the stimulus, and the reference homodyne wavefront as recovered by the CCD camera (red trace). The blue trace shows the heterodyne wavefront at the beginning of the stimulus (beginning of the ramp) as recovered from the different detectors, and 

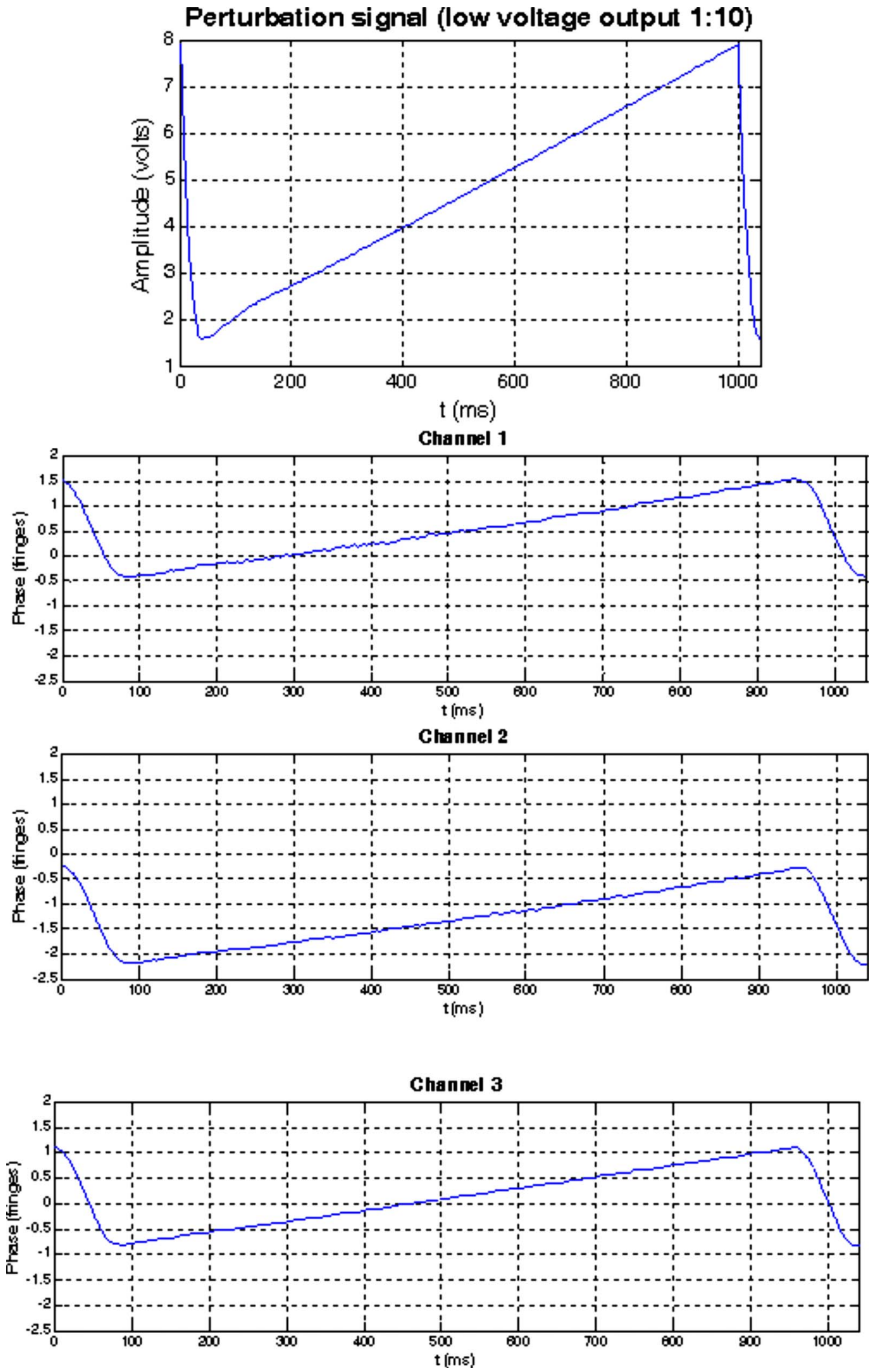

FIG. 3. (Color online) Temporal evolution of the heterodyne interferometric signals under a stimulus. Top trace PZT bias signal. Lower traces: channels 1-3.

the green trace is the heteroyne phasefront at the end of the stimulus (top of the ramp, see top trace of Fig. 3). In Fig. 4 we can see that the spatial profile remains invariant during the stimulus (blue and green traces) as the PZT introduces a displacement perpendicular to the propagation direction of the beam.
These results show in first place that we are able to perturb the common reference arm for the heterodyne/ homodyne interferometer and recover the temporal and spatial distribution for the phase for both. It is also shown that the spatial profile obtained for the heterodyne and homodyne interferometer follow the same pattern as the only variations 


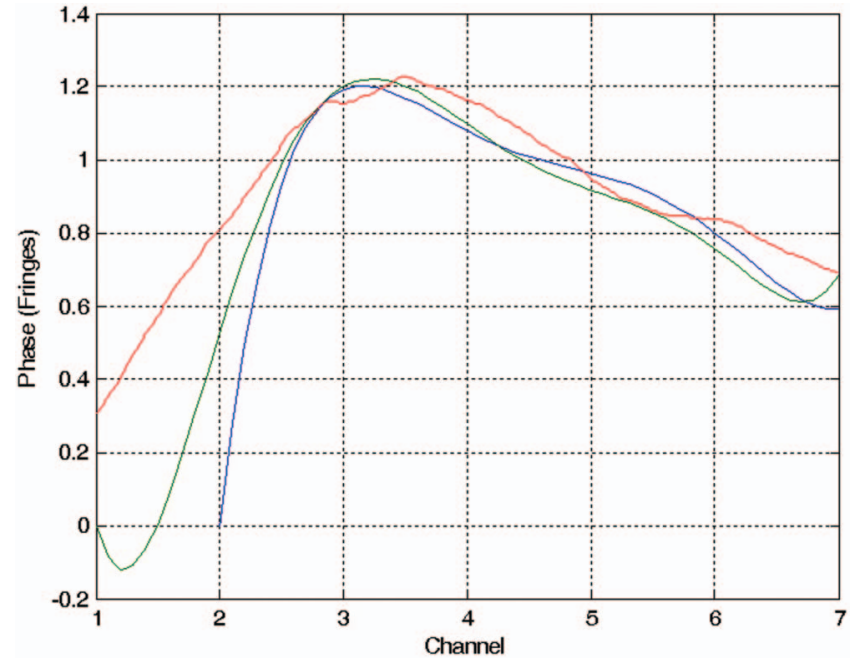

FIG. 4. (Color) Interferometric wavefronts recovered. Blue trace: heterodyne wavefront at the beginning of the stimulus. Green trace: heterodyne wavefront at the end of the stimulus. Red trace: reference homodyne wavefront from Fig. 2 (horizontal line).

in the spatial and longitudinal pathlength are introduced in the common arm (see Fig. 1). In the following paragraph this heterodyne/homodyne interferometer is used to evaluate the spatial resolution in the spatial profile reconstruction for the heterodyne interferometer.

\section{CALIBRATION OF THE SPATIAL RESOLUTION IN THE RECONSTRUCTION OF THE INTERFEROMETRIC HETERODYNE WAVEFRONT USING THE HETERODYNE/HOMODYNE INTERFEROMETER}

The optical setup described has been used also to calibrate directly the spatial resolution in the reconstruction of interferometric heterodyne wavefronts from the different discrete phase measurements obtained from each photodiode (Fig. 3) using the continuous intensity pattern profile from the homodyne system and taking advantage of the common

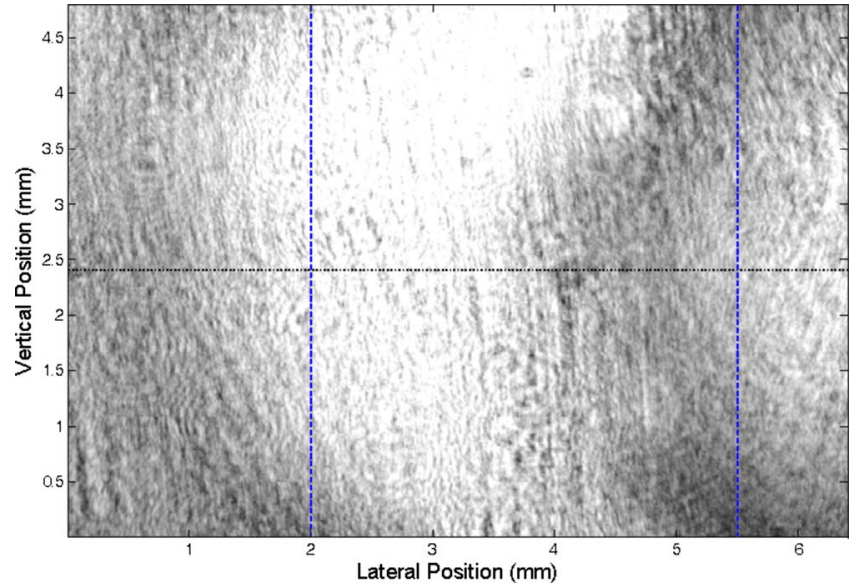

FIG. 5. (Color online) Homodyne interferometric pattern as recovered with the CCD camera for a several fringes spatial profile. Horizontal line: projection of the photodiode array for the heterodyne system. Vertical lines: area under study in Fig. 6.

measurement arm. This direct method described below allows a better estimation of the resolution than the indirect methods (for example, the use of Teflon sheets, Ref. 6) as both profiles are directly compared and no influence of diffraction is present.

For this purpose, we study a phase profile with more than one fringe phase difference along the interferometric wavefront introducing a slight misalignment in the common measurement arm of the heterodyne/homodyne interferometer. This is shown in Fig. 5 where the homodyne interferometric output acquired by the CCD camera presents two fringes in the spatial profile. In Fig. 6 we show the reference phasefront (the phase along the horizontal line in Fig. 5, red line) compared to the reconstructed heterodyne interferometric profile (black line). Note also in this figure the discrete phase measurements obtained from each of the array detectors with $1 \mathrm{~mm}$ center separation (dots) used to obtain the heterodyne phasefront. As we can see in Fig. 6 there is a

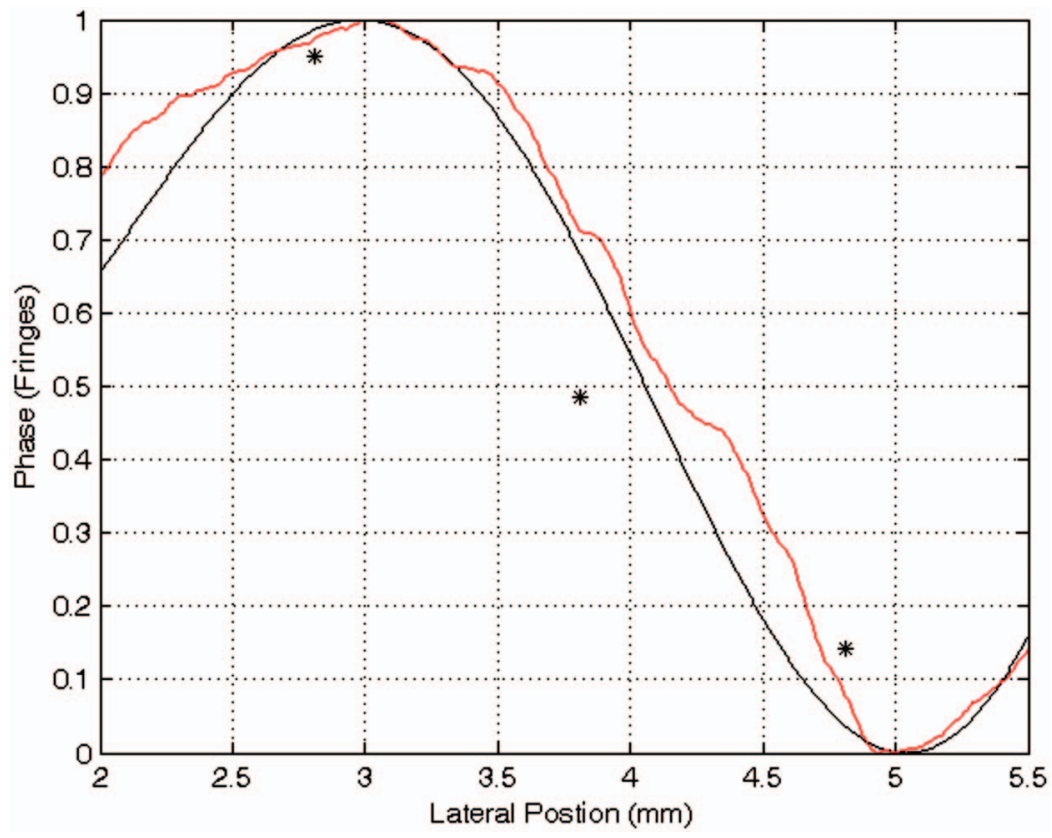

FIG. 6. (Color) Calibration of the heterodyne interferometric wavefront. Red trace: homodyne reference interferometric profile (Fig. 5 between vertical lines). Black trace: heterodyne wavefront recovered. Dots: phase measurement for each photodiode array element $(1 \mathrm{~mm}$ center separation). 
good match between the phase profiles recovered by both systems demonstrating a spatial resolution in the reconstruction of the heterodyne spatial profile better than $2 \mathrm{~mm}$.

These studies to validate the measurements and algorithm for the reconstruction of the heterodyne interferometric profile through comparison with the homodyne profile and the subsequent spatial resolution validation can be extended introducing more complex phase objects in the common measurement arm due to the versatility of this optical setup. Our next objectives include the study of the influence of different divergence angles, windows and other optical components to be included in the final diagnostic for the stellarator TJ-II. In this sense it is important to note that the already measured spatial resolution in the reconstruction of the profile in the $2 \mathrm{~mm}$ level meets the desired range for the stellarator TJ-II diagnostic objectives.
${ }^{1}$ D. R. Baker and S.-T. Lee, Rev. Sci. Instrum. 49, 919 (1978)

${ }^{2}$ J. H. Irby, E. S. Marmar, E. Sevillano, and S. M. Wolfe, Rev. Sci. Instrum. 59, 1568 (1988).

${ }^{3}$ P. Acedo, H. Lamela, M. Sánchez, T. Estrada, and J. Sánchez, Rev. Sci. Instrum. 75, 4671 (2004).

${ }^{4}$ T. N. Carlstrom, D. R. Ahlgren, and J. Crosbie, Rev. Sci. Instrum. 59, 1063 (1988)

${ }^{5}$ J. Irby, R. Murray, P. Acedo, and H. Lamela, Rev. Sci. Instrum. 70, 699 (1999)

${ }^{6}$ K. Tanaka, L. N. Vyacheslavov, K. Kawahata, T. Tokuzawa, and S. Okajima, Rev. Sci. Instrum. 72, 1089 (2001).

${ }^{7}$ K. Tanaka, A. L. Sanin, L. N. Vyacheslavov, Y. Akiyama, K. Kawahata, Y. Ito, and S. Okajima, Rev. Sci. Instrum. 75, 3429 (2004).

${ }^{8}$ C. Alejaldre, J. Alonso, J. botija, F. Castejón, J. R. Cepero, J. Guasp, A. López-Fraguas, L. García, V. I. Krivenski, R. Martín, A. P. Navarro, A. Perea, A. Rodríguez-Yunta, M. Sorolla, and A. Varías, Fusion Technol. 17, 131 (1990).

${ }^{9}$ M. Sánchez, J. Sánchez, T. Estrada, E. Sánchez, P. Acedo, and H. Lamela, Rev. Sci. Instrum. 75, 3414 (2004).

${ }^{10}$ H. Lamela, P. Acedo, and J. Irby, Rev. Sci. Instrum. 72, 96 (2001). 